\title{
Collaborative Multi-agent IVUS Image Segmentation
}

\author{
E.G.P. Bovenkamp, J. Dijkstra, J.G. Bosch, and J.H.C. Reiber \\ Leiden University Medical Center, Department of Radiology, \\ Building 1 C3-Q-50, Division of Image Processing, P.O. Box 9600, \\ 2300 RC Leiden, The Netherlands \\ E.G.P.Bovenkamp@lumc.nl
}

\begin{abstract}
This paper describes a multi-agent segmentation approach in which asynchronous agents combine low-level image processing with high-level reasoning. Agents each have their own image processing specialty and communicate and collaborate with other agents to resolve conflicts with the mutual goal to come to a consistent overall interpretation of image runs. Agents locally adjust their behavior and control the image processing depending on image content.
\end{abstract}

\section{Introduction}

A typical problem encountered in efforts to automate the image interpretation process is that there is, generally speaking, no 'ultimate segmentation algorithm'. Therefore an approach is desired, which deals with combining results from different segmentation algorithms, selecting the best solution given the image evidence and possibly following multiple lines of reasoning. A more elaborate and explicit reasoning system may handle such problems and lead to a successful interpretation of these images.

\section{System Design}

A multi-agent approach was chosen to enable modularization of knowledge and allow for scalability and flexibility. Further a multi-agent approach almost naturally fits the requirements that the system should be able to deal with combinations of different segmentation algorithms and multiple lines of reasoning. We designed and implemented a multi-agent system which integrates our locally developed image processing platform with the Soar architecture where Soar is an architecture for constructing general intelligent systems.

Agents in the system can interact with other agents through communication, act on the world by performing image processing operations and perceive that same world by accessing images and image processing results.

We have designed and implemented an image processing agent knowledge model that currently contains over 300 rules. Most of these rules represent common knowledge for each agent in the architecture and describe how to do image 
processing (139 rules), how to communicate (33 rules), how to resolve conflicts (100 rules) and further general utilities and problem solving (26 rules).

On average only about 5 extra rules are necessary to determine an agent's specialization. These rules specify an agents capabilities and interests and specify how an agent is to build complex image processing tasks. Less that $2 \%$ of the agent's knowledge is thus agent specific. This makes the knowledge model of image processing agents highly modular resulting in easy construction and addition of new agents to the agent community.

\section{Conflict Resolution}

Agent conflict resolution is the process in which agents try to achieve consensus over their image interpretations in case there is a conflict. A conflict arises when two or more agents hold incompatible views on the segmentation of images or when an agent detects internal inconsistencies. The conflict resolution mechanism is designed as a separate category of problem solving expertise.

The conflict resolution mechanism causes local adaptation to image content, combines low-level image processing knowledge with high-level knowledge about relations between objects given the global interpretation context, and enforces global consistency of the image segmentation.

\section{Results}

Table 1 shows the qualitative interpretation results of the collaborative multiagent image segmentation of 32 consecutive IntraVascular UltraSound (IVUS) images with 32 sidebranches and 13 shadows. The results compare the stand alone (sa) application of detection algorithms with their multi-agent application (ma). Optimal means a result matches segmentation by a human observer, suboptimal means acceptable but not perfect, and false means the result is not acceptable The table shows that no errors remain after conflict resolution (12 times) and algorithm adjustments (53 times) in the multi-agent approach, which illustrates its superior performance over stand alone application.

Table 1. Interpretation results of collaborative multi-agent IVUS image segmentation.

\begin{tabular}{lcccccccc}
\hline result & \multicolumn{2}{c}{ lumen } & \multicolumn{2}{c}{ vessel } & \multicolumn{2}{c}{ sidebranch } & \multicolumn{2}{c}{ shadow } \\
& sa & ma & sa & ma & sa & ma & sa & ma \\
\hline \hline optimal & 30 & 30 & 18 & 27 & 26 & 32 & 12 & 12 \\
suboptimal & 2 & 2 & 2 & 5 & 0 & 0 & 1 & 1 \\
false & 0 & 0 & 12 & 0 & 6 & 0 & 10 & 0 \\
\hline
\end{tabular}

\section{Acknowledgement}

This work has been funded by the Dutch Innovative Research Program (IOP) under grant nr. IBV97008. 